\title{
POSSIBLE CONTRIBUTIONS OF CLASSICAL ASTROMETRIC INSTRUMENT TO THE STUDY OF GEOSCIENCE AND REQUIREMENTS ON STAR CATALOGUES
}

\author{
HAN YANBEN and LI ZHISEN \\ Beijing Astronomical Observatory \\ Chinese Academy of Sciences \\ Beijing 100080 \\ CHINA
}

\begin{abstract}
In this paper possible contributions of classical instrument of time and latitude determinations in the study of geoscience is discussed. Some research results indicate that the observations of the classical instruments contain rich information on geoscience study. They can play a certain role in research of crust structure, plate motion, vertical variation, topography of core-mantle boundary, and in study of seismicity and major earthquake forecasting. It is important that a stable reference system is established and maintained and a relevant observed programme is formulated upon a star catalogue with good quality for geoscience and astro-geodynamics. Some instruments with high precision, especially located in seismic regions, would be continuously operated after the end of their task in ERP determination.
\end{abstract}

\section{Introduction}

For a long time, large contributions have been made to the time and latitude services and the study of catalogues by the classical astrometric observations and researches. The precision of the classical data was progressively improved along with improvements of the instruments and data processing. Now the mission of the classical instruments in determination of ERP will be replaced by new techniques with higher precision, such as VLBI, LLR and SLR, etc. The relating of the space reference system and terrestrial reference frame will still need the classical observations. Futhermore, can the classical instruments make more contributions?

Even if the more early classical observations were not concerned, the history of their systematic and extensive observation is near one hundrend years, and a wealth of data were accumulated. A result of the ILS in a homogeneous system (1899.9-1979.0) was given by Yumi[1], and astronomers have done a lot of work with the data. Li Zhengxin re-sorted out the data of BIH from 1962 to 1982 and provided a new evaluation[2]. Li's result showed that the development of the classical astrometry went through a period of great prosperity from 1960 's to 1970's and there were so much data. The new techniques can provide timely ERP with high precision, but their series are short. As it is often in the case of astrometry and geophysics, many scientists are very interested in the long series data in their researches. So the classical observations of different systems (e.g. ILS, BIH and IPMS, etc.) are seemingly 
still valuable. On the other side, the datum line of the classical instrument is the local vertical, the observations were inevitably affected by some geophysical factors. Although these factors have caused many troubles in the improvement of ERP determination, they set up the close ties between classical astrometry and geoscience. The study of some geoscientific subjects with the classical observations become feasible.

\section{Possible Contributions of Classical Instruments to Geoscience Research}

The continuous variations of local vertical reflect the fine structure of geoid surface. Developments of modern topography and gravimetry set a still higher demand on the determination of the vertical variation. A concept of the vertical deviation was continued to use in geodesy, it is different between astronomical coordinates and geodetic ones and reflects the deviation between the geoid and reference ellipsoid. The classical instruments measure the variation of angle between the local vertical and the Earth's axis, and the axis can be measured exactly by other methods (e.g. new techniques), therefore, the determination of vertical variation with the classical instruments is a quasi-absolute at least. Mironov has pointed out the possibility that the vertical variation was determinated by classical instruments[3]. Melchior has introduced the related works[4].

When the horizontal components of the variations in the gravitational field are about $0.2 \mathrm{mgal}$, the estimate of related variations of residuals $R T g$ in time and $R F g$ in latitude will be about 0.003 and 0 ".04 respectively. According to the present precision of classical instruments, their observations may reflect the long term vertical variation.

Classical data may also play a role in research and forecasting of major earthquakes. On 28 July 1976 , a major earthquke $(M=7.8)$ occurred in Tangshan region of China. An anomaly appearing in the observations of Danjon Astrolabe installed at Shahe Station of the Beijing Observatory about $160 \mathrm{~km}$ away from the epicentre was found by Zhang [6], and $\mathrm{Li}$ et al. [7]. Through the analysis of many major earthquakes and data of the $R T$ (or $O-A$ ) and $R F$, They pointed out that the interrelationship between the short-term anomalies of the $R T$ and $R F$ and the major earthquakes which occurred in regions around the classical instruments was confirmed. The relationships between $R F$ and earthquakes have been investigated also by others[8,9]. Over thirty major earthquakes which occurred in the regions around ten instruments have been studied, the observations of other instruments situated in places without major earthquakes were used for contrast. The results may be summarized as the following:

(1) The anomalies usually become apparent simultaneosly or separately in the $R T$ and $R F$ of the classical instrument before the occurrence of a major earthquake of $M \geq 7.0$ within the distant of $300 \mathrm{~km}$, or $M \geq 6.0$ within the distant of $100 \mathrm{~km}$; (2) The double peaks in different direction usually appear in $R T$ and only single peak in $R F$; (3) The beginning time of the anomalies were several weeks or months before the occurrence of the earthquake; (4) The vertical variation due to the motion of groundwater probably before the earthquake was considered as the main cause of the anomalies[6,10]; (5) The fluctuations of residuals of some instruments installed in the regions where no major earthquakes were relatively smaller.

Han et al. have studied the possibility that the information of earthquake forecasting was provided by classical instruments, and concluded that the instrument with high precision 
and observations can make the contribution to the major earthquake forecasting[11]. The result has been tested and verified by the photoelectrical astrolabe (PA) Mark-2 of Yunnan Observatory of China before a major earthquake[12].

Another area in which classical observations may play a role is the detection of plate motion. The motion of the Earth's plate would result in the long drift of observatory site, the information of which may be contained in the observation residuals of ERP. In 1960 's, the theory of plate tectonics was developed. After then, attempts were made to monitor the plate motion with classical instruments, but satisfactory results haven't been obtained, because of the limitation of measuring precision and the processing method of the observations[13].

The recent studies of some authors show broad prospects in this field. Li Zhengxin [14] gave one new method of total solution for reduction of the observations of 136 classical instruments in the whole world for 1962-1982.

New techniques have more precision, but with shorter history. It is important and helpful for the study of plate motion to use jointly the observations in different approaches.

\section{Requirements to Star Catalogue and Observational Program}

Due to the positive role of the classical instruments in the work of star catalogue, they will be retained to observe continously. As mentioned above, the classical instruments can make contributions to geoscience, and the new techniques cannot replace them completely. Therefore, the instruments can play double task of observing star catalogue and providing data for geoscience study simultaneously.

Since the geophysical effects usually are smaller except the anomalous refraction and vertical variation sometimes are larger, only a lot of observations with high precision are more significant for the geoscience study. The following points should be paid attention to:

(1) The chain observing of several groups will be achieved in every clear night as far as possible; (2) In order to ensure internal precision of a group, there should be enough stars in each group; (3) In order to reduce affection of change of meteorological condition and as far as possible to observe complete group or near complete one at short clear night, time interval owned by a group should be shorter; (4) In order to reduce effects of change of instrument constant and refraction, it is necessary that stars situated at different azimuth should be observed alternately for the astrolabe; (5) The program should be used continuously in longer period. This is important for ensured long-term stability of observations. Effects of precession and proper motion must be considered carefully.

Therefore, we need a catalogue with high quality in which precision is higher (position precision is better than \pm 0 " 05 ), so that the catalogue errors are reduced and we can compile a suitable program which can play double task in longer period. At present, the position precisions of bright stars of $F K 5$ may be satisfactory, but the stars are not so many.

\section{Concluding Remarks}

The positive role of classical instruments in geoscience study has initially been manifested, and their importance will be embodied by more detailed works. Acoording to the comparison and analysing of the data, astrolabe is more suitable for geoscience study among the classical instruments. Some astrolabes have been used for geoscience study in USA, USSR, 
DDR, and Japan, and a lot of interesting results have been obtained. Chinese Mark-2 PA have been modified in automation and photo-counting detector will be adopted. The precision and magnitude of star (up to $9.5 \mathrm{mag}$.) will be raised. Mark-3 PA will observe fainter stars (11mag.). It is necessary that classical instruments joint intimately with new techniques and geoscience instruments, and astronomers cooperate closely with geophysicists. Then more contributions of the classical instruments will be seen in the offing.

\section{References}

[1] Yumi, S. and Koyoyama, K. (1980), Results of the international latitude series in a homogeneous system 1899.9-1979.0, P167-172, Mizusawa.

[2] Li Zhengxin (1986), New determination of the Earth rotation parameters from optical astrometry observations, 1962.0-1982.0, Shanghai Technique and Science Press, Shanghai, China, (in Chinese).

[3] Mironov, N. T. et al. (1974), 'On the relative displacements of zenith of astronomical observatories', Proc. Geod. and Phys. Earth (2nd Inter. Symp. Geod. and Phys. Earth, May, 1973), pp. 173-181.

[4] Melchior, P. (1978), The tides of the planet Earth, Pergamon Press.

[5] Ma Zongjin et al. (1982), Nine major Earthquakes of China (1966-1976), Seismic Press, Beijing, (in Chinese).

[6] Zhang Guodong (1981), 'Deviation of the vertical caused by change of ground water level before a strong earthquake', Acta Seismologica Sinica, 3, No. 2, (in Chinese).

[7] Li Zhisen, Zhang Guodong et al. (1978), 'Correlation between the short anomalies of residuals astronomical time and latitude and the major earthquakes around the observatories', Acta Geophysica Sinica, 21, No. 4, pp. 278-290, (in Chinese).

[8] Mavliakov, G. A. et al. (1980), 'Anomalous variation of latitude and warning sign of the major earthquake of Alays mountain', J. Uzbekistan Geology, No. 2, pp. 66-70.

[9] Han Yanben et al. (1987), 'Occurrence of short-period anomaly of residuals of astronomical time-latitude at Yunnan Observatory preceding the Luquan Earthquake $\left(M_{L}=6.3\right)$ ', Kexue Tongbao (Science Bulletin), 32, No. 17, (in Chinese).

[10] Li Zhisen and Han Yanben, (1988), 'A possible geophysical interpretation of the anomalies in the residuals of astronomical time and latitude determination', Vistas in Astronomy, 31, pp. 671-675.

[11] Han Yanben et al. (1987), 'Possible warning sign of major earthquake in the observations of astronomical time and latitude', Publ. Beijing Astron. Observ., No. 10.

[12] Li Zhisen, (1988), 'Anomalous phenomena of time-latitude residual before earthquakes directly verified at Yunnan Astronomical Observatory', Recent Developments in World Seismology, No. 11, pp. 38-39, (in Chinese).

[13] Proverbio, E. F. et al. (1975), 'Astronomical evidence of change in the rate of the Earth's rotation and continental motion', In Growth Rhythms and History of the Earth's Rotation, (ed. Runcorn), pp. 385-395.

[14] Li Zhengxin (1989), 'Current plate motions from astrometry and Doppler Satellite observations', Acta Geophysica Sinica, 32, No. 5, pp. 567-573, (in Chinese). 